\title{
Mean-Square Asymptotically Almost Automorphic Solutions to Fractional Stochastic Relaxation Equations
}

\author{
Qiong Wu \\ Department of Mathematics, Tufts University, 503 Boston Avenue, Medford, MA 02155, USA \\ Correspondence should be addressed to Qiong Wu; qiong.wu@tufts.edu
}

Received 15 July 2015; Accepted 10 September 2015

Academic Editor: Yuji Liu

Copyright (C) 2015 Qiong Wu. This is an open access article distributed under the Creative Commons Attribution License, which permits unrestricted use, distribution, and reproduction in any medium, provided the original work is properly cited.

Mild solutions generated by a $(a, k)$-regularized family to fractional stochastic relaxation equations are studied. The main objective is to establish the existence and uniqueness of square-mean asymptotically almost automorphic mild solutions to linear and semilinear case of these equations. Under different hypotheses, some new theorems concerning the main objective are derived.

\section{Introduction}

Since the concept of almost automorphicity was introduced by Bochner [1], the automorphic functions have been applied to many areas such as ordinary as well as partial differential equations, abstract differential equations, functional differential equations, and integral equations; see, for example, [2-6]. For the details of basic theory and applications of almost automorphic functions, we refer the reader to the monographs of N'Guérékata [7,8]. In the early eighties, N'Guérékata introduced in [9] the concept of asymptotically almost automorphic functions; that is, functions that tend to an almost automorphic function at infinity, which is obviously a generalization of almost automorphicity. Since then, this function has been developed and applied extensively; see, for example, [10-14]. Recently, pseudoalmost automorphic functions were introduced by Liang et al. in [15] and they are more general than asymptotically almost automorphic functions. They have been studied and developed extensively, especially after the establishment of completeness of the space of vector-valued pseudoalmost automorphic functions under the supremum norm in [16]. For more details on these functions, we refer the reader to [17-19]. Due to the importance and applications of these functions in physics, mechanics, and mathematical biology, the study of existence of almost automorphic, asymptotically almost automorphic, and pseudoalmost automorphic solutions is becoming a very attractive topic in the qualitative theory of differential equations.
For the last few decades, time-fractional differential equations have appeared as an essential tool for studying dynamics of various complex processes on anomalous phenomena in physics, finance, hydrology, and cell biology; see [20-23]. The importance of using fractional methods in physics for describing slow decay processes and processes intermediate between relaxation and oscillation was stressed by Nigmatullin [24] in 1984. Various physical aspects of fractional relaxation were discussed in $[25,26]$. On the other hand, the topic of almost automorphic solutions to the fractional differential equations has also attracted a lot of attention, such as in $[27,28]$.

Recently, there has been an increasing interest in extending certain classical deterministic results to stochastic cases. The most important reason for such a consideration is that most problems in real life are inevitably subject to some random environmental effects. Thus, stochastic models are more natural and realistic to describe phenomena in the natural science; see [29]. In a very recent paper [30], the authors extended the almost automorphic function to its corresponding square-mean almost automorphic stochastic process. Also the mean-square asymptotically and pseudoalmost automorphic process were considered in the latter. For more details on these stochastic processes, we refer the reader to [31-34] and the references therein. However, to the best of the author's knowledge, there is no literature regarding mean-square asymptotically almost automorphic solutions for fractional stochastic relaxation equations. Such equations 
associated with a regularized resolvent family are important in fluid dynamics; see $[35,36]$.

In this paper, we investigate the existence and uniqueness of square-mean asymptotically almost automorphic solutions to the fractional stochastic relaxation equations of the linear form

$$
\begin{aligned}
& \mathrm{d} u(t)-\left(A \mathrm{D}_{t}^{\alpha} u(t)-u(t)\right) \mathrm{d} t \\
& \quad=f(t) \mathrm{d} t+g(t) \mathrm{d} W(t), \quad t>0,0<\alpha<1, \\
& u(0)=x
\end{aligned}
$$

and of the semilinear form

$$
\begin{aligned}
& \mathrm{d} u(t)-\left(A \mathrm{D}_{t}^{\alpha} u(t)-u(t)\right) \mathrm{d} t \\
& \quad=F(t, u(t)) \mathrm{d} t+G(t, u(t)) \mathrm{d} W(t), \\
& \quad t>0,0<\alpha<1, \\
& u(0)=x,
\end{aligned}
$$

where $u(\cdot)$ takes values in a separable Hilbert space $H, A$ : $D(A) \subset \mathscr{L}^{2}(\mathbb{P}, H) \rightarrow \mathscr{L}^{2}(\mathbb{P}, H)$ is a linear densely defined operator which is to be specified later, and $W(t)$ is a two-sided standard one-dimensional Brownian motion defined on the filtered probability space $\left(\Omega, \mathscr{F}, \mathbb{P}, \mathscr{F}_{t}\right) . \mathscr{F}_{t}=$ $\sigma\{W(u)-W(v) ; u, v \leq t\}$ is the natural filtration generated by the Brownian motion and $\mathbb{P}$ is the underlying probability measure. Here $f, g, F, G$ are appropriate functions to be specified later.

The paper is organized as follows. In Section 2, we briefly introduce some basic notations, definitions, and lemmas which are important for the proof of our main result. Section 3 presents our main results concerning existence and uniqueness of square-mean asymptotically almost automorphic solutions to a linear and semilinear case. Two different kinds of conditions for the linear and semilinear case are considered. Section 4 concludes the work in this paper and introduces the related work in progress.

\section{Preliminaries and Basic Properties}

Throughout this paper, we assume that $(H,\|\cdot\|)$ is a real separable Hilbert space, $(\Omega, \mathscr{F}, \mathbb{P})$ is a probability space, and $\mathscr{L}^{2}(\mathbb{P}, H)$ is the space of all $H$-valued random variables $x$ such that, for all $x \in \mathscr{L}^{2}(\mathbb{P}, H)$, we have the expected value $\mathbb{E}\|x\|^{2}=\int_{\Omega}\|x\|^{2} \mathrm{~d} \mathbb{P}<\infty$. So $\mathscr{L}^{2}(\mathbb{P}, H)$ is a Banach space equipped with the norm $\|x\|_{\mathscr{L}^{2}}=\left(\int_{\Omega}\|x\|^{2} \mathrm{~d} \mathbb{P}\right)^{1 / 2}$.

We denote by $C_{0}\left(\mathbb{R}^{+}, \mathscr{L}^{2}(\mathbb{P}, H)\right)$ the space of all continuous functions $h: \mathbb{R}^{+} \mapsto \mathscr{L}^{2}(\mathbb{P}, H)$ such that $\lim _{t \rightarrow \infty} \mathbb{E}\|h(t)\|^{2}=0$, and by $C_{0}\left(\mathbb{R}^{+} \times \mathscr{L}^{2}(\mathbb{P}, H), \mathscr{L}^{2}(\mathbb{P}, H)\right)$ the space of all continuous functions $h: \mathbb{R}^{+} \times \mathscr{L}^{2}(\mathbb{P}, H) \mapsto$ $\mathscr{L}^{2}(\mathbb{P}, H)$ such that $\lim _{t \rightarrow \infty} \mathbb{E}\|h(t, x)\|^{2}=0$ uniformly for $x$ in any compact subset of $\mathscr{L}^{2}(\mathbb{P}, H)$.

Definition 1. A stochastic process $x: \mathbb{R} \mapsto \mathscr{L}^{2}(\mathbb{P}, H)$ is said to be mean-square continuous or continuous in $\mathscr{L}^{2}(\mathbb{P}, H)$ if

$$
\lim _{t \rightarrow s} \mathbb{E}\|x(t)-x(s)\|^{2}=0
$$

Definition 2. A mean-square continuous process $x: \mathbb{R} \mapsto$ $\mathscr{L}^{2}(\mathbb{P}, H)$ is said to be square-mean almost automorphic (mean-square is defined by taking the mean of square of an object; square-mean is misnomer since it indicates taking square of the mean. However, I have retained it in paper due to the fact that the terminology is used in all primer papers on this subject) if, for every sequence of real numbers $\left(s_{n}^{\prime}\right)_{n \in \mathrm{N}}$, there is a subsequence $\left(s_{n}\right)_{n \in \mathrm{N}}$ and a stochastic process $y$ : $\mathbb{R} \mapsto \mathscr{L}^{2}(\mathbb{P}, H)$ such that

$$
\begin{aligned}
& \lim _{n \rightarrow \infty} \mathbb{E}\left\|x\left(t+s_{n}\right)-y(t)\right\|^{2}=0, \\
& \lim _{n \rightarrow \infty} \mathbb{E}\left\|y\left(t-s_{n}\right)-x(t)\right\|^{2}=0
\end{aligned}
$$

for each $t \in \mathbb{R}$.

Let $A A\left(\mathbb{R}, \mathscr{L}^{2}(\mathbb{P}, H)\right)$ be the collection of all square-mean almost automorphic stochastic processes. There are several properties of the space $A A\left(\mathbb{R}, \mathscr{L}^{2}(\mathbb{P}, H)\right)$.

Lemma 3 (see [30]). If $x, x_{1}$, and $x_{2}$ are all square-mean almost automorphic stochastic processes, then

(1) $x_{1}+x_{2}$ is square-mean almost automorphic;

(2) $\lambda x$ is square-mean almost automorphic for every scalar $\lambda$;

(3) there exists a constant $M>0$ such that $\sup _{t \in \mathbb{R}}\|x(t)\|_{\mathscr{L}^{2}} \leq M$. That is, $x$ is bounded in $\mathscr{L}^{2}(\mathbb{P}, H)$.

Lemma 4 (see $[30]) . A A\left(\mathbb{R}, \mathscr{L}^{2}(\mathbb{P}, H)\right)$ is a Banach space when it is equipped with the norm

$$
\|x\|_{\infty}:=\sup _{t \in \mathbb{R}}\left(\mathbb{E}\|x(t)\|^{2}\right)^{1 / 2},
$$

for $x \in A A\left(\mathbb{R}, \mathscr{L}^{2}(\mathbb{P}, H)\right)$.

Definition 5. A function $f: \mathbb{R} \times \mathscr{L}^{2}(\mathbb{P}, H) \mapsto \mathscr{L}^{2}(\mathbb{P}, H)$, $(t, x) \mapsto f(t, x)$, which is jointly continuous, is said to be square-mean almost automorphic in $t \in \mathbb{R}$ for each $x \in$ $\mathscr{L}^{2}(\mathbb{P}, H)$ if, for every sequence of real numbers $\left\{s_{n}^{\prime}\right\}_{n \in \mathrm{N}}$, there exists a subsequence $\left\{s_{n}\right\}$ such that, for some function $\widetilde{f}$, the following hold:

$$
\begin{aligned}
& \lim _{n \rightarrow \infty} \mathbb{E}\left\|f\left(t+s_{n}, x\right)-\tilde{f}(t, x)\right\|^{2}=0, \\
& \lim _{n \rightarrow \infty} \mathbb{E}\left\|\tilde{f}\left(t-s_{n}, x\right)-f(t, x)\right\|^{2}=0
\end{aligned}
$$

for each $t \in \mathbb{R}$ and each $x \in \mathscr{L}^{2}(\mathbb{P}, H)$.

Lemma 6 (see [30]). Let $f: \mathbb{R} \times \mathscr{L}^{2}(\mathbb{P}, H) \mapsto \mathscr{L}^{2}(\mathbb{P}, H)$, $(t, x) \mapsto f(t, x)$ be square-mean almost automorphic in $t$ for each $x \in \mathscr{L}^{2}(\mathbb{P}, H)$ and assume that $f$ satisfies a Lipschitz condition in the following sense:

$$
\mathbb{E}\|f(t, x)-f(t, y)\|^{2} \leq L \mathbb{E}\|x-y\|^{2}
$$


for all $x, y \in \mathscr{L}^{2}(\mathbb{P}, H)$ and for $t \in \mathbb{R}$, where $L>0$ is independent of $t$. Then, for any square-mean almost automorphic process $x: \mathbb{R} \mapsto \mathscr{L}^{2}(\mathbb{P}, H)$, the stochastic process $F$ : $\mathbb{R} \mapsto \mathscr{L}^{2}(\mathbb{P}, H)$ given by $F(t):=f(t, x(t))$ is square-mean almost automorphic.

Definition 7. A continuous function $f: \mathbb{R}^{+} \mapsto \mathscr{L}^{2}(\mathbb{P}, H)$ is said to be square-mean asymptotically almost automorphic if it can be written as $f=g+h$, where $g \in A A\left(\mathbb{R}, \mathscr{L}^{2}(\mathbb{P}, H)\right)$ and $h \in C_{0}\left(\mathbb{R}^{+}, \mathscr{L}^{2}(\mathbb{P}, H)\right)$. Let $A A A\left(\mathbb{R}^{+}, \mathscr{L}^{2}(\mathbb{P}, H)\right)$ denote the collection of all such functions.

Definition 8. A continuous function $f: \mathbb{R}^{+} \times \mathscr{L}^{2}(\mathbb{P}, H) \rightarrow$ $\mathscr{L}^{2}(\mathbb{P}, H)$ is said to be square-mean asymptotically almost automorphic in $t$ uniformly for $x$ in compact subsets of $\mathscr{L}^{2}(\mathbb{P}, H)$ if it can be written as $f=g+h$, where $g \in A A(\mathbb{R} \times$ $\left.\mathscr{L}^{2}(\mathbb{P}, H), \mathscr{L}^{2}(\mathbb{P}, H)\right)$ and $h \in C_{0}\left(\mathbb{R}^{+} \times \mathscr{L}^{2}(\mathbb{P}, H), \mathscr{L}^{2}(\mathbb{P}, H)\right)$. Let $A A A\left(\mathbb{R}^{+} \times \mathscr{L}^{2}(\mathbb{P}, H), \mathscr{L}^{2}(\mathbb{P}, H)\right)$ denote the collection of all such functions.

Let $K \subset \mathscr{L}^{2}(\mathbb{P}, H)$ and $Y \subset \mathbb{R}$. Also let $C_{K}(Y \times$ $\left.\mathscr{L}^{2}(\mathbb{P}, H), \mathscr{L}^{2}(\mathbb{P}, H)\right)$ denote the collection of all such functions $f: Y \times \mathscr{L}^{2}(\mathbb{P}, H) \rightarrow \mathscr{L}^{2}(\mathbb{P}, H)$ satisfying the condition that $f(t, \cdot)$ is uniformly continuous on $\mathscr{L}^{2}(\mathbb{P}, H)$ uniformly for $t \in Y$. The following properties concern the spaces $A A A\left(\mathbb{R}^{+}, \mathscr{L}^{2}(\mathbb{P}, H)\right)$ and $A A A\left(\mathbb{R}^{+} \times \mathscr{L}^{2}(\mathbb{P}, H), \mathscr{L}^{2}(\mathbb{P}, H)\right)$.

Lemma 9 (see [34]). $A A A\left(\mathbb{R}^{+}, \mathscr{L}^{2}(\mathbb{P}, H)\right)$ is a Banach space with the norm

$$
\|f\|_{\infty}:=\sup _{t \in \mathbb{R}^{+}}\left(\mathbb{E}\|f(t)\|^{2}\right)^{1 / 2},
$$

for $f \in A A A\left(\mathbb{R}^{+}, \mathscr{L}^{2}(\mathbb{P}, H)\right)$.

Lemma 10 (see [34]). Let $x \in A A A\left(\mathbb{R}^{+}, \mathscr{L}^{2}(\mathbb{P}, H)\right)$ and $f \in A A A\left(\mathbb{R}^{+} \times \mathscr{L}^{2}(\mathbb{P}, H), \mathscr{L}^{2}(\mathbb{P}, H)\right) \cap C_{K}\left(\mathbb{R}^{+} \times \mathscr{L}^{2}(\mathbb{P}\right.$, $\left.H), \mathscr{L}^{2}(\mathbb{P}, H)\right)$ with $K=\overline{\left\{x(t): t \in \mathbb{R}^{+}\right\}}$. Then $f(\cdot, x(\cdot)) \in$ $A A A\left(\mathbb{R}^{+}, \mathscr{L}^{2}(\mathbb{P}, H)\right)$.

Definition 11. Let $X$ be a Banach space; $k \in C\left(\mathbb{R}^{+}\right), k \neq 0$, and let $a \in \mathscr{L}_{\text {loc }}^{1}\left(\mathbb{R}^{+}\right), a \neq 0$. Assume that $A$ is a linear operator with domain $D(A)$. A strongly continuous family $\{R(t)\}_{t>0} \subset$ $\mathscr{B}(X)$ of bounded linear operators from $X$ into $X$ is called an $(a, k)$-regularized resolvent family on $X$ (or simply $(a, k)$ regularized family) having $A$ as a generator if the following hold:

(1) $R(0)=k(0) I$.

(2) $R(t) x \in D(A)$ and $R(t) A x=A R(t) x$ for all $x \in D(A)$ and $t \geq 0$.

(3) $R(t) x=k(t) x+\int_{0}^{t} a(t-s) A R(s) x \mathrm{~d} s, t \geq 0, x \in D(A)$.

\section{Main Results}

In this section, based on the definitions and lemmas given in the previous section, we derive conditions for existence and uniqueness of square-mean asymptotically almost automorphic mild solutions to fractional stochastic relaxation equations (1) and (2).

3.1. Linear Fractional Stochastic Relaxation Equation. To begin with, we consider linear fractional stochastic relaxation equation (1) where $A$ is the generator of a $(a, k)$-regularized family $R(t)$ with $k(t)=\exp (-t)$ and $a(t)=t^{\alpha} \mathbb{E}_{1,1-\alpha}(-t)$. Here $\mathbb{E}_{\alpha, \beta}(z)$ is the Mittag-Leffler function.

Definition 12. An $\mathscr{F}_{t}$-progressively measurable process $\{u(t)\}_{t \in \mathbb{R}^{+}}$is called a mild solution to (1) if it satisfies the following stochastic integral equation:

$$
\begin{aligned}
u(t)= & x-\int_{0}^{t} R(s) x \mathrm{~d} s+\int_{0}^{t} R(t-s) f(s) \mathrm{d} s \\
& +\int_{0}^{t} R(t-s) g(s) \mathrm{d} W_{s} .
\end{aligned}
$$

In order to get main result in this section, we need the following lemmas.

Lemma 13. Let $\{R(t)\}_{t \geq 0}$ be the $(a, k)$-regularized family generated by $A$ of (1) and $x \in \operatorname{Ker}(A)$, which is the kernel of the operator $A$. Then the function $I(t)$ over $t>0$ defined by

$$
I(t)=x-\int_{0}^{t} R(s) x \mathrm{~d} s
$$

is an element of $C_{0}\left(\mathbb{R}^{+}, \mathscr{L}^{2}(\mathbb{P}, H)\right)$.

Proof. Since $x \in \operatorname{Ker}(A)$, by Definition 11, we have

$$
\begin{aligned}
\mathbb{E}\|I(t)\|^{2} & =\mathbb{E}\left\|x-\int_{0}^{t} \exp (-s) x \mathrm{~d} s\right\|^{2} \\
& \leq\left|1-\int_{0}^{t} \exp (-s) \mathrm{d} s\right|^{2} \mathbb{E}\|x\|^{2} \\
& =\exp (-t) \mathbb{E}\|x\|^{2},
\end{aligned}
$$

which shows that $\lim _{t \rightarrow \infty} \mathbb{E}\|I(t)\|^{2}=0$ and this completes the proof.

Lemma 14. Let $\{R(t)\}_{t \geq 0}$ be the $(a, k)$-regularized family generated by $A$ of (1) and let $f(t), g(t)$ be $\operatorname{Ker}(A)$-valued square-mean asymptotically almost automorphic functions; then functions $F(t)$ and $G(t)$ defined by

$$
\begin{aligned}
& F(t)=\int_{0}^{t} R(t-s) f(s) \mathrm{d} s, \\
& G(t)=\int_{0}^{t} R(t-s) g(s) \mathrm{d} W_{s}
\end{aligned}
$$

are square-mean asymptotically almost automorphic; that is, $F(t), G(t) \in A A A\left(\mathbb{R}^{+}, \mathscr{L}^{2}(\mathbb{P}, H)\right)$.

Proof. First, we claim that $F(t) \in A A A\left(\mathbb{R}^{+}, \mathscr{L}^{2}(\mathbb{P}, H)\right)$. Obviously, $F(t)$ is continuous by definition. Since $f(s)$ is 
a $\operatorname{Ker}(A)$-valued function, we have $R(s) f(s)=\exp (-s) f(s)$ for all $s>0$. Moreover, $f(s) \in A A A\left(\mathbb{R}^{+}, \mathscr{L}^{2}(\mathbb{P}, H)\right)$, and thus there exist $f_{1}(s) \in A A\left(\mathbb{R}, \mathscr{L}^{2}(\mathbb{P}, H)\right)$ and $f_{2} \in$ $C_{0}\left(\mathbb{R}^{+}, \mathscr{L}^{2}(\mathbb{P}, H)\right)$ such that $f(s)=f_{1}(s)+f_{2}(s)$. Thus, we note that

$$
\begin{aligned}
F(t)= & \int_{0}^{t} R(t-s) f_{1}(s) \mathrm{d} s+\int_{0}^{t} R(t-s) f_{2}(s) \mathrm{d} s \\
= & \int_{-\infty}^{t} R(t-s) f_{1}(s) \mathrm{d} s-\int_{-\infty}^{0} R(t-s) f_{1}(s) \mathrm{d} s \\
& +\int_{0}^{t} R(t-s) f_{2}(s) \mathrm{d} s .
\end{aligned}
$$

We now show that $F_{1}(t) \in A A\left(\mathbb{R}, \mathscr{L}^{2}(\mathbb{P}, H)\right)$. Since $f_{1}(s) \in$ $A A\left(\mathbb{R}, \mathscr{L}^{2}(\mathbb{P}, H)\right)$, for every real sequence $\left\{s_{n}^{\prime}\right\}_{n \in \mathrm{N}}$, there exist a subsequence $\left\{s_{n}\right\}_{n \in \mathrm{N}}$ and a function $\tilde{f}_{1}(s): \mathbb{R} \rightarrow \mathscr{L}^{2}(\mathbb{P}, H)$ such that, for all $t \in \mathbb{R}$,

$$
\begin{aligned}
& \lim _{n \rightarrow \infty} \mathbb{E}\left\|f_{1}\left(s+s_{n}\right)-\tilde{f}_{1}(s)\right\|^{2}=0, \\
& \lim _{n \rightarrow \infty} \mathbb{E}\left\|\tilde{f}_{1}\left(s-s_{n}\right)-f_{1}(s)\right\|^{2}=0 .
\end{aligned}
$$

On the other hand, let

$$
\widetilde{F}_{1}(t)=\int_{-\infty}^{t} R(t-s) \tilde{f}_{1}(s) \mathrm{d} s
$$

Then, it holds

$$
\begin{aligned}
& \mathbb{E}\left\|F_{1}\left(t+s_{n}\right)-\widetilde{F}_{1}(t)\right\|^{2}=\mathbb{E} \| \int_{-\infty}^{t+s_{n}} R\left(t+s_{n}-s\right) \\
& \cdot f_{1}(s) \mathrm{d} s-\int_{-\infty}^{t} R(t-s) \widetilde{f}_{1}(s) \mathrm{d} s \|^{2} \\
& =\mathbb{E} \| \int_{-\infty}^{t} R(t-s) f_{1}\left(s+s_{n}\right) \mathrm{d} s-\int_{-\infty}^{t} R(t-s) \\
& \cdot \tilde{f}_{1}(s) \mathrm{d} s\left\|^{2}=\mathbb{E}\right\| \int_{-\infty}^{t} \exp (-(t-s)) \\
& \cdot f_{1}\left(s+s_{n}\right) \mathrm{d} s-\int_{-\infty}^{t} \exp (-(t-s)) \tilde{f}_{1}(s) \mathrm{d} s \|^{2}
\end{aligned}
$$

$$
\begin{aligned}
& \leq \mathbb{E}\left(\int_{-\infty}^{t} \exp (-(t-s))\right. \\
& \left.\cdot\left\|f_{1}\left(s+s_{n}\right)-\tilde{f}_{1}(s)\right\| \mathrm{d} s\right)^{2} \leq \int_{-\infty}^{t} \exp (-(t-s)) \\
& \cdot \mathbb{E}\left\|f_{1}\left(s+s_{n}\right)-\tilde{f}_{1}(s)\right\|^{2} \mathrm{~d} s
\end{aligned}
$$

which shows that $\lim _{n \rightarrow \infty} \mathbb{E}\left\|F_{1}\left(t+s_{n}\right)-\widetilde{F}_{1}(t)\right\|^{2}=0$.

Similarly, $\lim _{n \rightarrow \infty} \mathbb{E}\left\|\widetilde{F}_{1}\left(t-s_{n}\right)-F_{1}(t)\right\|^{2}=0$ is obtained. Thus, $F_{1}(t) \in A A\left(\mathbb{R}, \mathscr{L}^{2}(\mathbb{P}, H)\right)$ is proved.

Next, we prove that $F_{2}(t) \in C_{0}\left(\mathbb{R}^{+}, \mathscr{L}^{2}(\mathbb{P}, H)\right)$. Since $f_{2}(s) \in C_{0}\left(\mathbb{R}^{+}, \mathscr{L}^{2}(\mathbb{P}, H)\right)$, for all $\epsilon>0$, there exists a constant $T>0$ such that, for all $s>2 T, \mathbb{E}\left\|f_{2}(s)\right\|^{2}<\epsilon$. Thus, for all $t \geq T$, we have

$$
\begin{aligned}
& \mathbb{E}\left\|F_{2}(t)\right\|^{2}=\mathbb{E} \| \int_{0}^{t / 2} R(t-s) f_{2}(s) \mathrm{d} s \\
& +\int_{t / 2}^{t} R(t-s) f_{2}(s) \mathrm{d} s-\int_{-\infty}^{0} R(t-s) f_{1}(s) \mathrm{d} s \|^{2} \\
& \leq 3 \mathbb{E}\left\|\int_{0}^{t / 2} R(t-s) f_{2}(s) \mathrm{d} s\right\|^{2} \\
& +3 \mathbb{E}\left\|\int_{t / 2}^{t} R(t-s) f_{2}(s) \mathrm{d} s\right\|^{2} \\
& +3 \mathbb{E}\left\|\int_{-\infty}^{0} R(t-s) f_{1}(s) \mathrm{d} s\right\|^{2} \\
& \leq 3 \int_{0}^{t / 2} \exp (-(t-s)) \mathrm{d} s \\
& \cdot \int_{0}^{t / 2} \exp (-(t-s)) \mathbb{E}\left\|f_{2}(s)\right\|^{2} \mathrm{~d} s \\
& +3 \int_{t / 2}^{t} \exp (-(t-s)) \mathrm{d} s \\
& \cdot \int_{t / 2}^{t} \exp (-(t-s)) \mathbb{E}\left\|f_{2}(s)\right\|^{2} \mathrm{~d} s \\
& +3 \int_{-\infty}^{0} \exp (-(t-s)) \mathrm{d} s \\
& \cdot \int_{-\infty}^{0} \exp (-(t-s)) \mathbb{E}\left\|f_{1}(s)\right\|^{2} \mathrm{~d} s \leq 3\left\|f_{2}\right\|_{\infty}^{2} \\
& \cdot \exp (-t)+3 \epsilon+3\left\|f_{1}\right\|_{\infty}^{2} \exp (-2 t),
\end{aligned}
$$

which indicates that $\lim _{t \rightarrow \infty} \mathbb{E}\left\|F_{2}(t)\right\|^{2}=0$ and this means that $F_{2}(t) \in C_{0}\left(\mathbb{R}^{+}, \mathscr{L}^{2}(\mathbb{P}, H)\right)$. Recalling that $F(t)=$ $F_{1}(t)+F_{2}(t)$ for all $t \geq 0$, we conclude that $F(t) \in$ $A A A\left(\mathbb{R}^{+}, \mathscr{L}^{2}(\mathbb{P}, H)\right)$.

There remains claiming that $G(t) \in A A A\left(\mathbb{R}^{+}, L^{2}(\mathbb{P}, H)\right)$. From its definition, we know that $G(t)$ is continuous. 
Also since $g(s) \in A A A\left(\mathbb{R}^{+}, \mathscr{L}^{2}(\mathbb{P}, H)\right)$, we have $g_{1}(s) \in$ $A A\left(\mathbb{R}, \mathscr{L}^{2}(\mathbb{P}, H)\right)$ and $g_{2}(t) \in C_{0}\left(\mathbb{R}^{+}, \mathscr{L}^{2}(\mathbb{P}, H)\right)$ such that $g(s)=g_{1}(s)+g_{2}(s)$. We observe that

$$
\begin{aligned}
G(t)= & \int_{0}^{t} R(t-s) g_{1}(s) \mathrm{d} W_{s} \\
& +\int_{0}^{t} R(t-s) g_{2}(s) \mathrm{d} W_{s} \\
= & \int_{-\infty}^{t} R(t-s) g_{1}(s) \mathrm{d} W_{s} \\
& -\int_{-\infty}^{0} R(t-s) g_{1}(s) \mathrm{d} W_{s} \\
& +\int_{0}^{t} R(t-s) g_{2}(s) \mathrm{d} W_{s} .
\end{aligned}
$$

Let

$$
\begin{aligned}
G_{1}(t)= & \int_{-\infty}^{t} R(t-s) g_{1}(s) \mathrm{d} W_{s}, \quad t \in \mathbb{R}, \\
G_{2}(t)= & -\int_{-\infty}^{0} R(t-s) g_{1}(s) \mathrm{d} W_{s} \\
& +\int_{0}^{t} R(t-s) g_{2}(s) \mathrm{d} W_{s}, \quad t \geq 0 .
\end{aligned}
$$

We first show that $G_{1}(t) \in A A\left(\mathbb{R}, \mathscr{L}^{2}(\mathbb{P}, H)\right)$. Since $g_{1}(t) \epsilon$ $A A\left(\mathbb{R}, \mathscr{L}^{2}(\mathbb{P}, H)\right)$, for every real sequence $\left\{s_{n}^{\prime}\right\}_{n \in \mathrm{N}}$, there exists subsequence $\left\{s_{n}\right\}_{n \in \mathrm{N}}$ and a function $\widetilde{g}_{1}: \mathbb{R} \rightarrow$ $\mathscr{L}^{2}(\mathbb{P}, H)$ such that, for all $t \in \mathbb{R}$,

$$
\begin{aligned}
& \lim _{n \rightarrow \infty} \mathbb{E}\left\|g_{1}\left(s+s_{n}\right)-\widetilde{g}_{1}(s)\right\|^{2}=0, \\
& \lim _{n \rightarrow \infty} \mathbb{E}\left\|\widetilde{g}_{1}\left(s-s_{n}\right)-g_{1}(s)\right\|^{2}=0 .
\end{aligned}
$$

Let

$$
\widetilde{G}_{1}(t)=\int_{-\infty}^{t} R(t-s) \widetilde{g}_{1}(s) \mathrm{d} W_{s} ;
$$

we obtain

$$
\begin{aligned}
\mathbb{E} & \left\|G_{1}\left(t+s_{n}\right)-\widetilde{G}_{1}(t)\right\|^{2} \\
& =\mathbb{E} \| \int_{-\infty}^{t+s_{n}} R\left(t+s_{n}-s\right) g_{1}(s) \mathrm{d} W_{s} \\
& -\int_{-\infty}^{t} R(t-s) \widetilde{g}_{1}(s) \mathrm{d} W_{s} \|^{2} \\
& =\mathbb{E} \| \int_{-\infty}^{t} R(t-s) g_{1}\left(s+s_{n}\right) \mathrm{d} \widetilde{W}_{s} \\
& -\int_{-\infty}^{t} R(t-s) \widetilde{g}_{1}(s) \mathrm{d} W_{s} \|^{2},
\end{aligned}
$$

where $\widetilde{W}_{s}=W\left(s+s_{n}\right)-W_{s_{n}}$ for each $s \in \mathbb{R}$ and $n \in \mathrm{N}$. We know that $\widetilde{W}_{s}$ is also a Brownian motion and has the same distribution as $W_{s}$. Thus, we get

$$
\begin{aligned}
\mathbb{E} & \left\|G_{1}\left(t+s_{n}\right)-\widetilde{G}_{1}(t)\right\|^{2} \\
& =\mathbb{E}\left\|\int_{-\infty}^{t} \exp (-(t-s))\left[g_{1}\left(s+s_{n}\right)-\widetilde{g}_{1}(s)\right] \mathrm{d} \widetilde{W}_{s}\right\|^{2} \\
& =\int_{\infty}^{t} \exp (-2(t-s)) \mathbb{E}\left\|g_{1}\left(s+s_{n}\right)-\widetilde{g}_{1}(s)\right\|^{2} \mathrm{~d} s,
\end{aligned}
$$

which implies that $\lim _{n \rightarrow \infty} \mathbb{E}\left\|G_{1}\left(t+s_{n}\right)-\widetilde{G}_{1}(t)\right\|^{2}=0$.

Analogously, $\lim _{n \rightarrow \infty} \mathbb{E}\left\|\widetilde{G}_{1}\left(t-s_{n}\right)-G_{1}(t)\right\|^{2}=0$. It means $G(t) \in A A\left(\mathbb{R}, \mathscr{L}^{2}(\mathbb{P}, H)\right)$.

Next, we show that $G_{2}(t) \in C_{0}\left(\mathbb{R}^{+}, \mathscr{L}^{2}(\mathbb{P}, H)\right)$. Since $g_{2}(s) \in C_{0}\left(\mathbb{R}^{+}, \mathscr{L}^{2}(\mathbb{P}, H)\right)$, for all $\epsilon>0$, there exists a constant $T>0$ such that, for all $s \geq T$, EE $\left\|g_{2}(s)\right\|^{2}<\epsilon$. Then, for all $t \geq 2 T$, we compute

$$
\begin{aligned}
& \mathbb{E}\left\|G_{2}(t)\right\|^{2}=\mathbb{E} \| \int_{0}^{t / 2} R(t-s) g_{2}(s) \mathrm{d} W_{s} \\
& +\int_{t / 2}^{t} R(t-s) g_{2}(s) \mathrm{d} W_{s} \\
& \quad-\int_{-\infty}^{0} R(t-s) g_{1}(s) \mathrm{d} W_{s} \|^{2} \\
& \quad \leq 3 \mathbb{E}\left\|\int_{0}^{t / 2} R(t-s) g_{2}(s) \mathrm{d} W_{s}\right\|^{2} \\
& +3 \mathbb{E}\left\|\int_{t / 2}^{t} R(t-s) g_{2}(s) \mathrm{d} W_{s}\right\|^{2} \\
& +3 \mathbb{E}\left\|\int_{-\infty}^{0} R(t-s) g_{1}(s) \mathrm{d} W_{s}\right\|^{2} \\
& \quad=3 \int_{0}^{t / 2} \exp (-2(t-s)) \mathbb{E}\left\|g_{2}(s)\right\|^{2} \mathrm{~d} s \\
& \quad+3 \int_{t / 2}^{t} \exp (-2(t-s)) \mathbb{E}\left\|g_{2}(s)\right\|^{2} \mathrm{~d} s \\
& \quad+3 \int_{-\infty}^{0} \exp (-2(t-s)) \mathbb{E}\left\|g_{1}(s)\right\|^{2} \mathrm{~d} s \\
& \quad \leq \frac{3}{2}\left\|g_{2}\right\|_{\infty}^{2} \exp (-t)+\frac{3}{2} \epsilon+\frac{3}{2}\left\|g_{1}\right\|_{\infty}^{2} \exp (-2 t),
\end{aligned}
$$

which implies that $\lim _{t \rightarrow \infty} \mathbb{E}\left\|G_{2}(t)\right\|^{2}=0$ and then $G_{2}(t) \epsilon$ $C_{0}\left(\mathbb{R}^{+}, \mathscr{L}^{2}(\mathbb{P}, H)\right)$. Therefore, $G(t) \in A A A\left(\mathbb{R}^{+}, \mathscr{L}^{2}(\mathbb{P}, H)\right)$ since $G(t)=G_{1}(t)+G_{2}(t)$. Thus the proof is complete.

The main result concerning linear fractional stochastic relaxation equation (1) is the following theorem.

Theorem 15. If $x \in \operatorname{Ker}(A)$ and $f(t), g(t)$ are $\operatorname{Ker}(A)$ valued square-mean asymptotically almost automorphic functions, then every mild solution of (1) is asymptotically almost automorphic. 
Proof. From Definition 12, we have the following mild solution to linear fractional stochastic relaxation equation (1):

$$
\begin{aligned}
u(t)= & x-\int_{0}^{t} R(s) x \mathrm{~d} s+\int_{0}^{t} R(t-s) f(s) \mathrm{d} s \\
& +\int_{0}^{t} R(t-s) g(s) \mathrm{d} W_{s} \\
= & I(t)+F_{1}(t)+G_{1}(t)+F_{2}(t)+G_{2}(t),
\end{aligned}
$$

where $I(t), F_{1}(t), F_{2}(t), G_{1}(t)$, and $G_{2}(t)$ are the expressions given in Lemmas 13 and 14. Then $u(t)$ has decomposition of $F_{1}(t)+G_{1}(t)$ and $I(t)+F_{2}(t)+G_{2}(t)$ which are in $A A\left(\mathbb{R}, \mathscr{L}^{2}(\mathbb{P}, H)\right)$ and $C_{0}\left(\mathbb{R}^{+}, \mathscr{L}^{2}(\mathbb{P}, H)\right)$, respectively. This completes the proof.

3.2. Semilinear Fractional Stochastic Relaxation Equations. In this section, we consider the existence and uniqueness of a square-mean asymptotically almost automorphic mild solution of (2) where $A$ has the same meaning as in the linear case.

Definition 16. An $\mathscr{F}_{t}$-progressively measurable process $\{u(t)\}_{t \in \mathbb{R}}$ is called a mild solution to (2) if it satisfies the following stochastic integral equation:

$$
\begin{aligned}
u(t)= & x-\int_{0}^{t} R(s) x \mathrm{~d} s+\int_{0}^{t} R(t-s) f(s, u(s)) \mathrm{d} s \\
& +\int_{0}^{t} R(t-s) g(s, u(s)) \mathrm{d} W_{s} .
\end{aligned}
$$

Here when we consider that $u(0)=x \in \operatorname{Ker}(A)$ and $f(\cdot, u(\cdot)), g(\cdot, u(\cdot))$ are $\operatorname{Ker}(A)$-valued square-mean asymptotically almost automorphic functions; the results derived in the last section can be generalized to semilinear case.

In order to establish the existence and uniqueness result for the semilinear case with more flexible initial conditions and coefficients, we need the following assumptions and lemma:

(1) There exists an $(a, k)$-regularized family such that $\|R(t)\| \leq M k(t)$ for all $t \geq 0$ and some constant $M>0$.

(2) The function $f \in A A A\left(\mathbb{R}^{+} \times \mathscr{L}^{2}(\mathbb{P}, H), \mathscr{L}^{2}(\mathbb{P}, H)\right)$ and there exists a function $L_{f}: \mathbb{R}^{+} \rightarrow \mathbb{R}^{+}$such that, for each $r \geq 0$ and $\mathbb{E}\|u\|^{2}, \mathbb{E}\|v\|^{2} \leq r$,

$$
\mathbb{E}\|f(t, u)-f(t, v)\|^{2} \leq L_{f}(r) \mathbb{E}\|u-v\|^{2} .
$$

(3) The function $g \in A A A\left(\mathbb{R}^{+} \times \mathscr{L}^{2}(\mathbb{P}, H), \mathscr{L}^{2}(\mathbb{P}, H)\right)$ and there exists a function $L_{g}: \mathbb{R}^{+} \rightarrow \mathbb{R}^{+}$such that, for each $r \geq 0$ and $\mathbb{E}\|u\|^{2}, \mathbb{E}\|v\|^{2} \leq r$,

$$
\mathbb{E}\|g(t, u)-g(t, v)\|^{2} \leq L_{g}(r) \mathbb{E}\|u-v\|^{2} .
$$

(4) $\Lambda:=\sup _{r>0}\left[r / 4 M^{2}-2 r L_{f}(r)-r L_{g}(r)\right]>(1+$ $1 / \mathrm{M})\|u(0)\|^{2}+2 \sup _{s \in \mathbb{R}} \mathbb{E}\|f(s, 0)\|^{2}+\sup _{s \in \mathbb{R}} \mathbb{E}\|g(s, 0)\|^{2}$.
Lemma 17. Let $\{R(t)\}_{t \geq 0}$ be the $(a, k)$-regularized family generated by $A$ of (2) and let assumption (1) hold. Then the function $I(t)$ defined by

$$
I(t)=M x-\int_{0}^{t} R(s) x \mathrm{~d} s \quad t>0
$$

is an element of $C_{0}\left(\mathbb{R}^{+}, \mathscr{L}^{2}(\mathbb{P}, H)\right)$.

Proof. Similar to Lemma 13, we have

$$
\begin{aligned}
\mathbb{E}\|I(t)\|^{2} & =\mathbb{E}\left\|M x-\int_{0}^{t} R(s) x \mathrm{~d} s\right\|^{2} \\
& \leq M\left|1-\int_{0}^{t} \exp (-s) \mathrm{d} s\right|^{2} \mathbb{E}\|x\|^{2} \\
& =M \exp (-t) \mathbb{E}\|x\|^{2},
\end{aligned}
$$

which shows that $\lim _{t \rightarrow \infty} \mathbb{E}\|I(t)\|^{2}=0$ and this completes the proof.

Theorem 18. Assume that the assumptions (1)-(4) hold. Then there exists a unique square-mean asymptotically almost automorphic mild solution to (2).

Proof. Let $D=\left\{u \in A A A\left(\mathbb{R}^{+}, \mathscr{L}^{2}(\mathbb{P}, H)\right): \mathbb{E}\|u\|^{2} \leq r\right\}$. Then $D$ is a closed subspace of $A A A\left(\mathbb{R}^{+}, \mathscr{L}^{2}(\mathbb{P}, H)\right)$. We define an operator $\Psi$ on $D$ by

$$
\begin{aligned}
(\Psi u)(t)= & x-\int_{0}^{t} R(s) \mathrm{d} s+\int_{0}^{t} R(t-s) f(s, u(s)) \mathrm{d} s \\
& +\int_{0}^{t} R(t-s) g(s, u(s)) \mathrm{d} W_{s}, \quad t \geq 0 .
\end{aligned}
$$

First, let us check the fact that $\Psi\left(A A A\left(\mathbb{R}^{+}, \mathscr{L}^{2}(\mathbb{P}, H)\right)\right) \subset$ $A A A\left(\mathbb{R}^{+}, \mathscr{L}^{2}(\mathbb{P}, H)\right)$. It is obvious that $\Psi u$ is continuous for $u \in A A A\left(\mathbb{R}^{+}, \mathscr{L}^{2}(\mathbb{P}, H)\right)$. Define $K=\overline{\left\{u(t): t \in \mathbb{R}^{+}\right\}}$. It follows from assumptions (2) and (3) that $f, g \in A A A\left(\mathbb{R}^{+} \times\right.$ $\left.\mathscr{L}^{2}(\mathbb{P}, H), \mathscr{L}^{2}(\mathbb{P}, H)\right) \cap C_{K}\left(\mathbb{R}^{+}, \mathscr{L}^{2}(\mathbb{P}, H)\right)$. From Lemma 10, we get $f(\cdot, u(\cdot))$ and $g(\cdot, u(\cdot)) \in A A A\left(\mathbb{R}^{+}, \mathscr{L}^{2}(\mathbb{P}, H)\right)$. Now, by Lemma 14, we have

$$
\begin{aligned}
F(t) & =\int_{0}^{t} R(t-s) f(s) \mathrm{d} s \in A A A\left(\mathbb{R}^{+}, \mathscr{L}^{2}(\mathbb{P}, H)\right), \\
G(t) & =\int_{0}^{t} R(t-s) g(s) \mathrm{d} W_{s} \\
& \in A A A\left(\mathbb{R}^{+}, \mathscr{L}^{2}(\mathbb{P}, H)\right) .
\end{aligned}
$$

Combining this with Lemma 17, we conclude that $\Psi u \in$ $A A A\left(\mathbb{R}^{+}, \mathscr{L}^{2}(\mathbb{P}, H)\right)$. Next we need to show that $\Psi$ is 
a contraction map from $D$ into $D$. We can take $u(t) \in D$ for all $t \geq 0$ and get

$$
\begin{aligned}
& \mathbb{E}\|(\Psi u)(t)\|^{2}=\mathbb{E} \| x-\int_{0}^{t} R(s) x \mathrm{~d} s \\
& +\int_{0}^{t} R(t-s) f(s, u(s)) \mathrm{d} s \\
& +\int_{0}^{t} R(t-s) g(s, u(s)) \mathrm{d} W_{s}\left\|^{2} \leq 4\right\| x \|^{2} \\
& +4\left\|\int_{0}^{t} R(s) x \mathrm{~d} s\right\|^{2} \\
& +4 \mathbb{E}\left(\int_{0}^{t} M \exp (-(t-s))\|f(s, u(s))\| \mathrm{d} s\right)^{2} \\
& +4 \mathbb{E} \int_{0}^{t} M^{2} \exp (-2(t-s))\|g(s, u(s))\|^{2} \mathrm{~d} s \\
& \quad \leq 4\|x\|^{2}+4 M^{2}\|x\|^{2}+8 M^{2} \int_{0}^{t} \exp (-(t-s)) \\
& +4\|x\|^{2}+4 M^{2}\|x\|^{2}+8 M^{2}(L+(r) r \\
& +\left(\mathbb{E}\|f(s, u(s))-f(s, 0)\|^{2}+\|f(s, 0)\|^{2}\right) \mathrm{d} s \\
& +8 M^{2} \int_{0}^{t} \exp (-2(t-s)) \\
& +\left(\mathbb{E}\|g(s, u(s))-g(s, 0)\|^{2}+\|g(s, 0)\|^{2}\right) \mathrm{d} s \\
& +4 M^{2}\left(L,(r) r+\|g(s, x)\|_{\infty}^{2}\right)<r
\end{aligned}
$$

where the last inequality is from assumption (4). This indicates that $\Psi u \in D$ for any given $u \in D$.

For $u, v \in D$ and $t \geq 0$, we have

$$
\begin{aligned}
& \mathbb{E}\|(\Psi u)(t)-(\Psi v)(t)\|^{2} \\
& \leq 2 \mathbb{E}\left\|\int_{0}^{t} R(t-s)(f(s, u(s))-f(s, v(s))) \mathrm{d} s\right\|^{2} \\
& \quad+2 \mathbb{E}\left\|\int_{0}^{t} R(t-s)(g(s, u(s))-g(s, v(s))) \mathrm{d} W_{s}\right\|^{2} \\
& \leq 2 M^{2} L_{f}(r)\|u-v\|_{\infty}^{2}+M^{2} L_{g}(r)\|u-v\|_{\infty}^{2} \\
& \leq\left(2 L_{f}(r)+L_{g}(r)\right) M^{2}\|u-v\|_{\infty}^{2} .
\end{aligned}
$$

Hence

$$
\begin{aligned}
& \|(\Psi u)(t)-(\Psi v)(t)\|_{\infty}^{2} \\
& \quad \leq\left(2 L_{f}(r)+L_{g}(r)\right) M^{2}\|u-v\|_{\infty}^{2} .
\end{aligned}
$$

From assumption (4), we know that there exists a $r$ such that

$$
\left(2 L_{f}(r)+L_{g}(r)\right) M^{2}<1 .
$$

This implies that $\Psi$ is a contraction map from $D$ into $D$. So by the Banach fixed point theorem, $\Psi$ has a unique fixed point in $D$, which indicates that there exists a square-mean asymptotically almost automorphic mild solution to (2).

\section{Conclusion}

In this paper, the square-mean asymptotically almost automorphic solutions to abstract fractional stochastic relaxation equations have been investigated. Based on $(a, k)$-regularized family of bounded and linear operators, mild solutions to fractional stochastic relaxation equations are derived by a variation of constant formula. By using stochastic analysis and the Banach fixed point theorem, a set of sufficient conditions are obtained to prove the existence and uniqueness of square-mean asymptotically almost automorphic solutions to fractional stochastic relaxation equations. My related investigation on the asymptotical behaviors of solutions to a class of fractional stochastic differential equations with nonlocal initial conditions is still in progress.

\section{Conflict of Interests}

The author declares that there is no conflict of interests regarding the publication of this paper.

\section{Acknowledgments}

The author wishes to thank Dr. Marjorie Hahn for her help and advice and the author's peer L. Chlebak for discussions and valuable suggestions after reading first version of this paper as well as Dr. Patricia Garmirian for discussions. The author would also like to thank the editor for his suggestion on the clarity of the paper.

\section{References}

[1] S. Bochner, "A new approach to almost periodicity," Proceedings of the National Academy of Sciences of the United States of America, vol. 48, no. 12, pp. 2039-2043, 1962.

[2] T. Diagana, G. Nguerekata, and N. V. Minh, "Almost automorphic solutions of evolution equations," Proceedings of the American Mathematical Society, vol. 132, no. 11, pp. 3289-3298, 2004.

[3] H.-S. Ding, T.-J. Xiao, and J. Liang, "Existence of positive almost automorphic solutions to nonlinear delay integral equations," Nonlinear Analysis: Theory, Methods \& Applications, vol. 70, no. 6, pp. 2216-2231, 2009.

[4] K. Ezzinbi and G. M. N’Guérékata, "Massera type theorem for almost automorphic solutions of functional differential equations of neutral type," Journal of Mathematical Analysis and Applications, vol. 316, no. 2, pp. 707-721, 2006.

[5] G. M. N’Guérékata, "Existence and uniqueness of almost automorphic mild solutions to some semilinear abstract differential equations," Semigroup Forum, vol. 69, no. 1, pp. 80-86, 2004.

[6] J. A. Goldstein and G. M. N’Guérékata, "Almost automorphic solutions of semilinear evolution equations," Proceedings of the American Mathematical Society, vol. 133, no. 8, pp. 2401-2408, 2005. 
[7] G. M. N'Guérékata, Almost Automorphic Functions and Almost Periodic Functions in Abstract Spaces, Kluwer Academic/Plenum, New York, NY, USA, 2001.

[8] G. M. N’Guérékata, Topics in Almost Automorphy, Springer, New York, NY, USA, 2005.

[9] G. M. N’Guérékata, “Sur les solutions presque automorphes dequations differentielles abstraites," Annales des Sciences Mathématiques du Québec, vol. 5, pp. 69-79, 1981.

[10] D. Bugajewski and G. M. N'Guérékata, “On the topological structure of almost automorphic and asymptotically almost automorphic solutions of differential and integral equations in abstract spaces," Nonlinear Analysis: Theory, Methods \& Applications, vol. 59, no. 8, pp. 1333-1345, 2004.

[11] H.-S. Ding, T.-J. Xiao, and J. Liang, "Asymptotically almost automorphic solutions for some integrodifferential equations with nonlocal initial conditions," Journal of Mathematical Analysis and Applications, vol. 338, no. 1, pp. 141-151, 2008.

[12] T. Diagana, M. E. Hernández, and J. P. C. dos Santos, "Existence of asymptotically almost automorphic solutions to some abstract partial neutral integro-differential equations," Nonlinear Analysis: Theory, Methods \& Applications, vol. 71, no. 1-2, pp. 248-257, 2009.

[13] A. Caicedo, C. Cuevas, G. M. Mophou, and G. M. N’Guérékata, "Asymptotic behavior of solutions of some semilinear functional differential and integro-differential equations with infinite delay in Banach spaces," Journal of the Franklin InstituteEngineering and Applied Mathematics, vol. 349, no. 1, pp. 1-24, 2012.

[14] J. P. C. dos Santos and C. Cuevas, "Asymptotically almost automorphic solutions of abstract fractional integro-differential neutral equations," Applied Mathematics Letters, vol. 23, no. 9, pp. 960-965, 2010.

[15] J. Liang, J. Zhang, and T.-J. Xiao, "Composition of pseudo almost automorphic and asymptotically almost automorphic functions," Journal of Mathematical Analysis and Applications, vol. 340, no. 2, pp. 1493-1499, 2008.

[16] T.-J. Xiao, J. Liang, and J. Zhang, "Pseudo-almost automorphic solutions to semilinear differential equations in Banach spaces," Semigroup Forum, vol. 76, no. 3, pp. 518-524, 2008.

[17] K. Ezzinbi, S. Fatajou, and G. M. N'guérékata, "Pseudo-almostautomorphic solutions to some neutral partial functional differential equations in Banach spaces," Nonlinear Analysis, Theory, Methods and Applications, vol. 70, no. 4, pp. 1641-1647, 2009.

[18] J. Liang, G. M. N'Guérékata, T.-J. Xiao, and J. Zhang, "Some properties of pseudo-almost automorphic functions and applications to abstract differential equations," Nonlinear Analysis, Theory, Methods \& Applications, vol. 70, no. 7, pp. 2731-2735, 2009.

[19] K. Ezzinbi, H. Toure, and I. Zabsonre, "Pseudo almost automorphic solutions of class $r$ for some partial functional differential equations," Afrika Matematika, vol. 25, no. 1, pp. 25-41, 2014.

[20] R. Metzler and J. Klafter, "The random walk's guide to anomalous diffusion: a fractional dynamics approach," Physics Reports, vol. 339, no. 1, pp. 1-77, 2000.

[21] J. Janczura and A. Wyłomańska, "Subdynamics of financial data from fractional Fokker-Planck equation," Acta Physica Polonica $B$, vol. 40, no. 5, pp. 1341-1351, 2009.

[22] D. A. Benson, S. W. Wheatcraft, and M. M. Meerschaert, "Application of a fractional advection-dispersion equation," Water Resources Research, vol. 36, no. 6, pp. 1403-1412, 2000.
[23] M. J. Saxton and K. Jacobson, "Single-particle tracking: applications to membrane dynamics," Annual Review of Biophysics and Biomolecular Structure, vol. 26, pp. 373-399, 1997.

[24] R. R. Nigmatullin, "On the theory of relaxation for systems with 'remnant' memory," Physica Status Solidi (B), vol. 124, no. 1, pp. 389-393, 1984.

[25] T. F. Nonnenmacher and W. G. Glöckle, "A fractional model for mechanical stress relaxation," Philosophical Magazine Letters, vol. 64, no. 2, pp. 89-93, 1991.

[26] A. Carpinteri and F. Mainardi, Fractals and Fractional Calculus in Continuum Mechanics, Springer, New York, NY, USA, 1997.

[27] D. Araya and C. Lizama, "Almost automorphic mild solutions to fractional differential equations," Nonlinear Analysis, Theory, Methods and Applications, vol. 69, no. 11, pp. 3692-3705, 2008.

[28] C. Cuevas and C. Lizama, "Almost automorphic solutions to a class of semilinear fractional differential equations," Applied Mathematics Letters, vol. 21, no. 12, pp. 1315-1319, 2008.

[29] B. K. Oksendal, Stochastic Differential Equations, Springer, Berlin, Germany, 2005.

[30] M. Fu and Z. Liu, "Square-mean almost automorphic solutions for some stochastic differential equations," Proceedings of the American Mathematical Society, vol. 138, no. 10, pp. 3689-3701, 2010.

[31] Z. Chen and W. Lin, "Square-mean pseudo almost automorphic process and its application to stochastic evolution equations," Journal of Functional Analysis, vol. 261, no. 1, pp. 69-89, 2011.

[32] Y.-K. Chang, Z.-H. Zhao, and G. M. N'Guérékata, "A new composition theorem for square-mean almost automorphic functions and applications to stochastic differential equations," Nonlinear Analysis, Theory, Methods \& Applications, vol. 74, no. 6, pp. 2210-2219, 2011.

[33] Y.-K. Chang, Z.-H. Zhao, and G. M. N'Gurkata, "Squaremean almost automorphic mild solutions to non-autonomous stochastic differential equations in Hilbert spaces," Computers and Mathematics with Applications, vol. 61, no. 2, pp. 384-391, 2011.

[34] Z. Yan and H.-W. Zhang, "Square-mean asymptotically almost automorphic solutions for nonlocal neutral stochastic functional integro-differential equations in Hilbert space," Electronic Journal of Mathematical Analysis and Applications, vol. 1, no. 1, pp. 15-30, 2013.

[35] C. Lizama and G. M. N'Guérékata, "Mild solutions for abstract fractional differential equations," Applicable Analysis, vol. 92, no. 8, pp. 1731-1754, 2013.

[36] A. Karczewska and C. Lizama, "Solutions to stochastic fractional relaxation equations," Physica Scripta, vol. 2009, no. T136, 2009. 


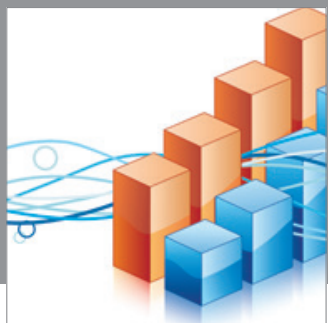

Advances in

Operations Research

mansans

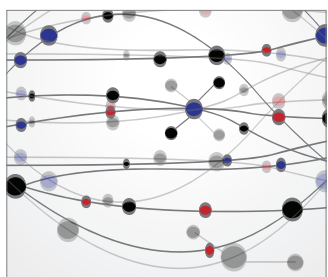

The Scientific World Journal
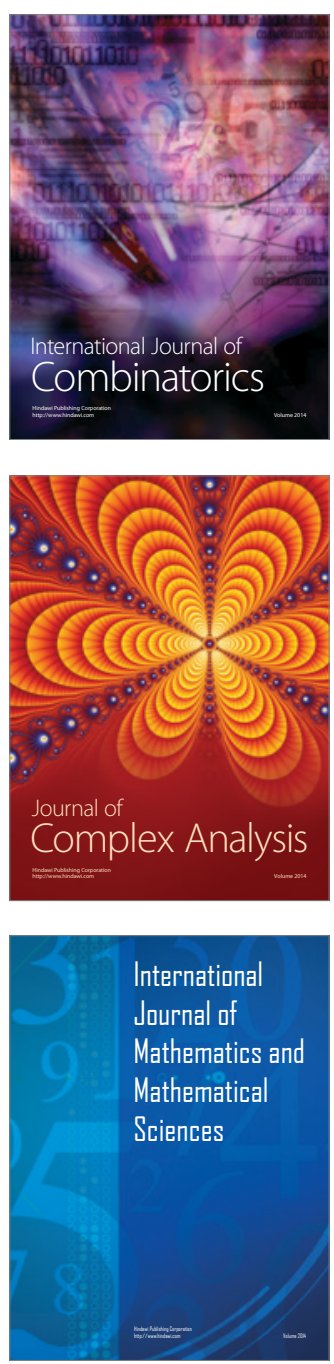
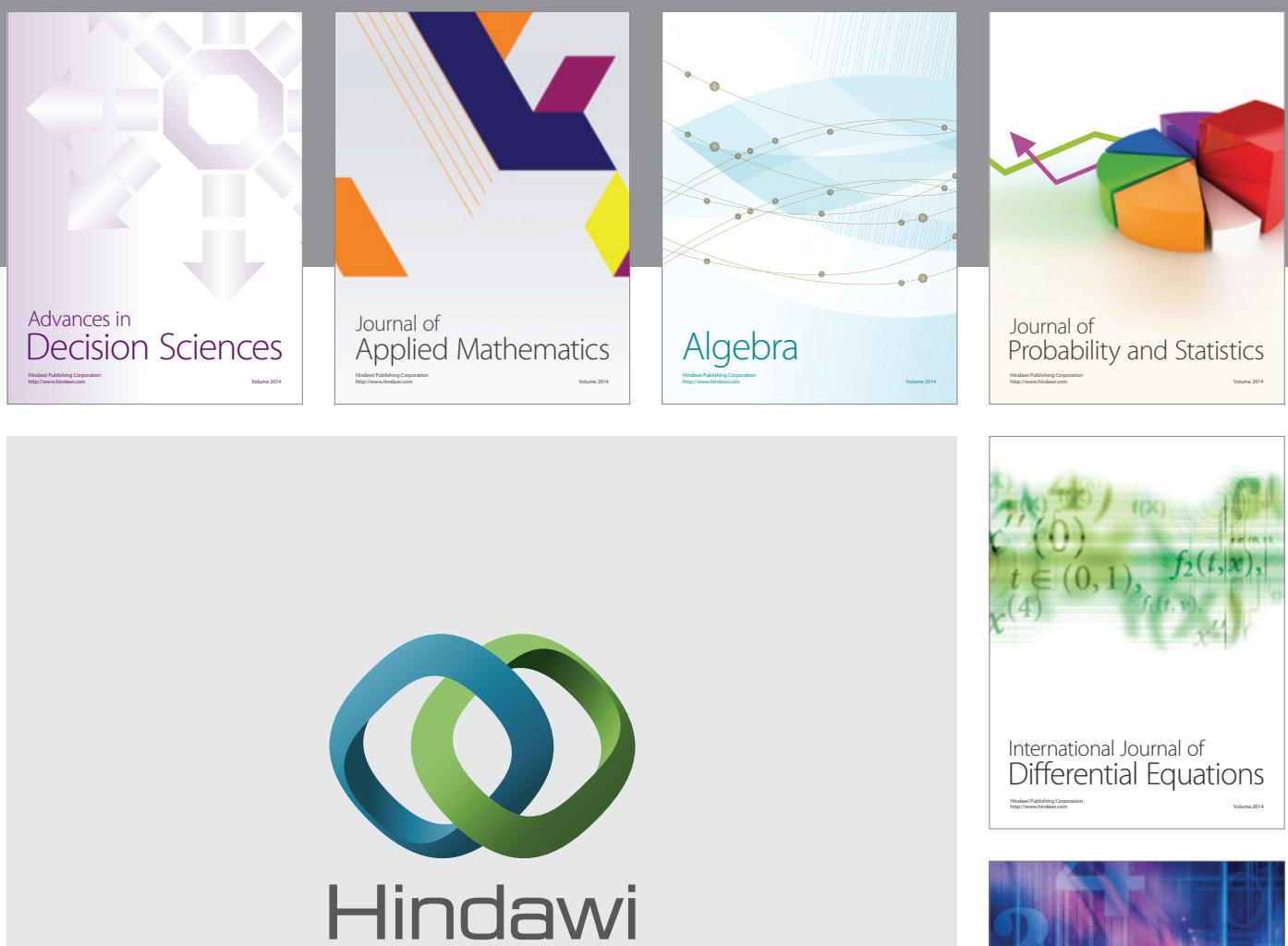

Submit your manuscripts at http://www.hindawi.com
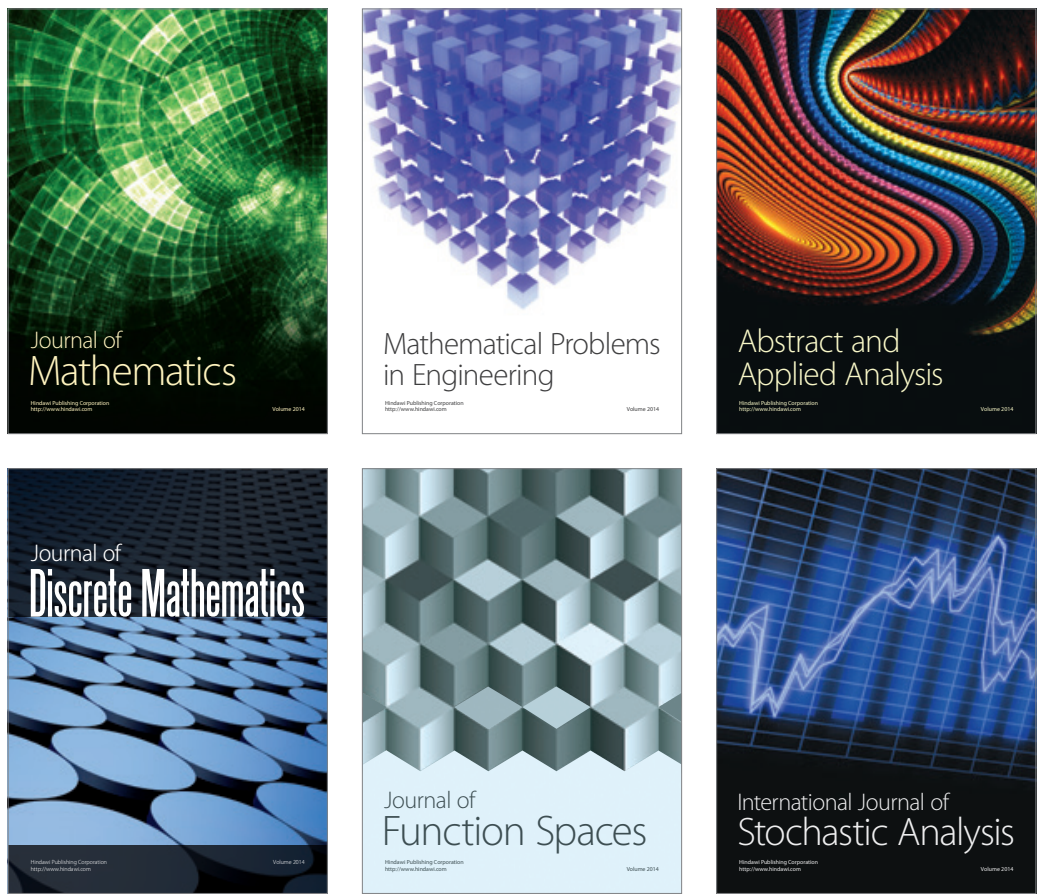

Journal of

Function Spaces

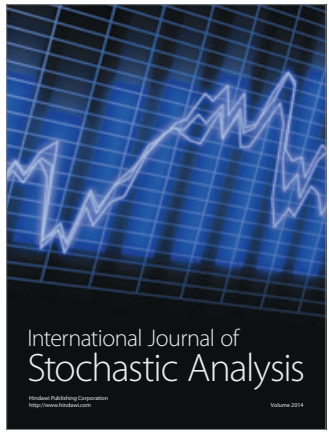

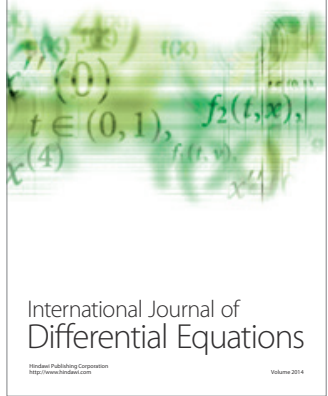
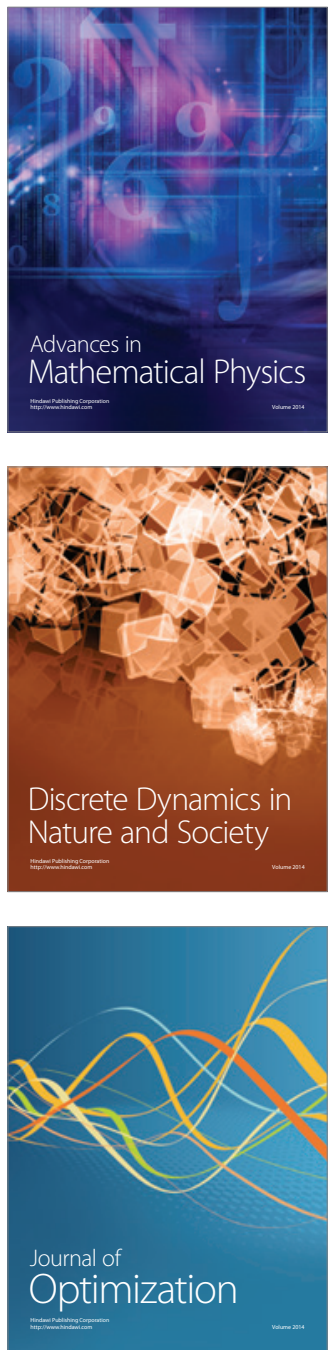